\title{
La FMH et le projet Helsana
}

Le groupe d'assurance Helsana a présenté il y a quelques semaines un modèle de fonctionnement qui ne manque pas d'intérêt, pour l'assurance-maladie obligatoire. A quelques nuances près, mais importantes, ce modèle reprend en fait ce que la FMH a proposé à la Commission de la santé du Conseil des Etats il y a deux ans! C'est a priori étonnant, mais c'est peut-être l'un des premiers résultats de la campagne du $1^{\mathrm{er}}$ juin?

Regardons cela d'un peu plus près. Notre système de santé ne représente pas un marché comme l'est le marché des produits industriels ou des services. La santé n'est en effet pas une denrée de consommation courante mais un bien existentiel pour chacun d'entre nous, qui est de surcroît ancré de façon très solidaire dans notre société. Notre système de santé s'est développé en parallèle avec la société; il en découle ainsi qu'heureusement le diagnostic médical et les soins ne dépendent pas premièrement de questions financières.

$\mathrm{Au}$ vu des défis qui l'attendent, comme l'évolution démographique, le développement de la médecine, et l'augmentation des maladies chroniques, cependant, nous sommes clairement appelés à gérer notre système de santé de la manière la plus économique. Un système de santé économique signifie pour le corps médical que tous les acteurs portent en commun le souci de travailler ensemble à un haut niveau de qualité, de façon économiquement tenable à long terme, et avec une préoccupation sociale. La FMH reconnaît au projet récemment diffusé par Helsana divers points positifs allant dans ce sens:

- l'obligation de contracter, autrement le libre choix du médecin, est maintenue dans l'assurance de base comme fondement de notre système de soins;

- le Managed Care est et reste un choix libre pour tous les partenaires du système de santé: patients, fournisseurs de prestations et assureurs;

- chaque caisse-maladie est dans l'obligation d'offrir des systèmes de réseau et de Managed Care;

- les incitations financières qui seront nouvellement proposées aux assurés font également de ceux-ci des partenaires actifs dans le système de santé.

Il faut relever en particulier dans le projet présenté par Helsana que le choix entre un modèle avec un libre-choix complet ou un modèle de Managed Care, est laissé entièrement à l'assuré.

Alors - car il y en a bien sûr! - quels sont les problèmes que pose le projet publié par Helsana? Nous en voyons quatre, de divers ordres:
- Le Managed Care ne peut évidemment pas fonctionner avec les seuls patients malades, qu'une franchise ou une participation réduites convaincraient d'adhérer; des incitations pour les assurés en bonne santé, au niveau des primes, sont également indispensables pour assurer aux réseaux une composition équilibrée.

- Les incitations financières prévues pour le modèle Managed Care ne devraient pas être telles qu'elle représente en réalité une limitation de la liberté de choix; incitation oui, mais dans le respect de toutes les situations socio-économiques, sans quoi le Managed Care deviendra une «médecine du pauvre», ce que nous ne voulons en aucun cas.

- Il est absolument essentiel que pour chaque réseau de Managed Care, un contrat existe entre un groupe de médecins et l'assureur; cette condition doit explicitement figurer dans la loi et nous nous battrons contre toute solution qui ne le prévoirait pas: nous ne voulons en aucun cas rester sous la menace des «modèles de listes» ou d'autre fantaisies unilatérales de certains assureurs! Seul un contrat peut garantir la juste reconnaissance des médecins comme partenaires.

- Et il doit s'agir de groupes de médecins et pas de contrats individuels: un médecin isolé ne dispose pas du nombre de patients nécessaire à produire des statistiques fiables, et n'a pas non plus le poids nécessaire à conduire des négociations équilibrées. De toute façon, le Managed Care, ce n'est pas un médecin isolé!

- Enfin, quatrième point, Il est nécessaire dans ces modèles de prévoir un suivi scientifique des soins; sans parler de la problématique de la compensation des risques, qui doit absolument être résolue.

C'est dans cette esprit, et en se battant pour que les améliorations indispensables soient prises en compte, que la FMH participera aux travaux de la Commission du Conseil des Etats cet été.

Nous ne sommes bien sûr pas opposés à l'évolution du système de santé, elle est nécessaire et doit tirer profit des propositions constructives qui sont faites - mais elle doit aussi se faire en garantissant que les progrès en soient vraiment et ne menacent pas le futur et la qualité de notre travail.

Dr Jacques de Haller, président de la FMH 